\title{
Números cromossômicos e implicações sistemáticas em espécies da subfamília Caesalpinioideae (Leguminosae) ocorrentes na região sul do Brasil
}

\author{
ELAINE BIONDO ${ }^{1}$, SILVIA TERESINHA S. MIOTTO ${ }^{2,4}$ e MARIA TERESA SCHIFINO-WITTMANN³
}

(recebido: 8 de abril de 2004; aceito: 10 de novembro de 2005)

\begin{abstract}
Chromosome numbers and systematic implications in species of subfamily Caesalpinioideae (Leguminosae) from the Southern region of Brazil). The subfamily Ceasalpinioideae (Leguminosae) comprises around 2,800 species, many of which occurring in Brazil. For the Southern region of Brazil, 56 species economically, socially and scientifically important, are cited, distributed along different environments, but detailed taxonomic and cytogenetic studies are still missing. This paper reports chromosome number analysis in 74 accessions of 27 taxa belonging to ten genera of tribes Cassieae, Caesalpinieae, and Cercideae. The determined chromosome numbers were $2 \mathrm{n}=32,28,26,24,22,16$ and 14 . For seven species the chromosome numbers were determined for the first time: Cassia leptophylla, Senna araucarietorum, S. hilariana, S. neglecta, S. oblongifolia, Chamaecrista repens and Pomaria stipularis. Most species had $2 \mathrm{n}=28$ chromosomes, $2 \mathrm{n}=26,24$, and 22 being also found. The genus Chamaecrista differs from the others studied as all its taxa presented $2 \mathrm{n}=32,16$, and 14 , the first one derived by polyploidy. The basic number of $\mathrm{x}=14$ was proposed for the species of nine of the studied genera, while $\mathrm{x}=13,12$ and 11 would have probably been originated by disploidy. For the genus Chamaecrista, $\mathrm{x}=8$ is suggested for most species and $\mathrm{x}=7$ for the species of section Xerocalyx. Polyploidy seems to have played an important role in the initial diversification of the group, accompanied by several disploid reductions during the evolutionary process. The number of chromosomes distinguishes Chamaecrista from the other genera. This, together with other analyzed characteristics, as well as literature data, supports the separation of this genus from the other Cassieae genera.
\end{abstract}

Key words - Caesalpinioideae, chromosome number, evolution, Leguminosae, systematics

RESUMO - (Números cromossômicos e implicações sistemáticas em espécies da subfamília Caesalpinioideae (Leguminosae) ocorrentes na região Sul do Brasil). A subfamília Caesalpinioideae (Leguminosae) possui cerca de 2.800 espécies, muitas das quais ocorrem no Brasil. Para a região Sul do Brasil são citadas 56 espécies, distribuídas pelos mais diversos ambientes, com importância econômica, social e científica bastante grande, sendo ainda pouco conhecidas do ponto de vista citogenético e taxonômico. Neste estudo foram analisados, quanto ao número de cromossomos, 74 acessos de 27 táxons incluídos em 10 gêneros pertencentes às tribos Cassieae, Caesalpinieae e Cercideae. Os números cromossômicos encontrados foram $2 \mathrm{n}=32$, 28, 26, 24, 22, 16 e 14. Sete espécies tiveram seus números cromossômicos determinados pela primeira vez: Cassia leptophylla, Senna araucarietorum, S. hilariana, S. neglecta, S. oblongifolia, Chamaecrista repens e Pomaria stipularis. A maioria das espécies apresentaram $2 \mathrm{n}=28$ cromossomos, sendo observados também $2 \mathrm{n}=26,24$ e 22 . O gênero Chamaecrista diferencia-se dos demais gêneros, pois todos os seus táxons apresentaram $2 n=32,16$ e 14 cromossomos, sendo o primeiro número supostamente originado por poliploidia. $\mathrm{O}$ número básico proposto para as espécies estudadas foi $\mathrm{x}=14$, com os demais números, $\mathrm{x}=13,12 \mathrm{e} 11$, tendo surgido provavelmente por disploidia e para o gênero Chamaecrista $\mathrm{x}=8$ e $\mathrm{x}=7$ para a espécie pertencente à seção Xerocalyx. A poliploidia pareceu importante na diversificação inicial do grupo, com ocorrência de uma série de reduções displóides no decorrer do processo evolutivo. O caráter número de cromossomos mostrou-se relevante na distinção de táxons do gênero Chamaecrista dos demais gêneros, sugerindo, juntamente com outros caracteres analisados e encontrados em literatura, a segregação deste dos demais gêneros pertencentes à tribo Cassieae.

Palavras-chave - Caesalpinioideae, evolução, Leguminosae, números cromossômicos, sistemática

1. Universidade Federal do Rio Grande do Sul, Instituto de Biociências, Departamento de Botânica, Programa de PósGraduação em Botânica, Av. Bento Gonçalves 9500, prédio 43433, Campus do Vale, 91501-970 Porto Alegre, RS, Brasil.

2. Universidade Federal do Rio Grande do Sul, Instituto de Biociências, Departamento de Botânica, Av. Bento Gonçalves 9500, prédio 43433, Campus do Vale, 91501-970 Porto Alegre, RS, Brasil.

3. Universidade Federal do Rio Grande do Sul, Faculdade de Agronomia, Departamento de Plantas Forrageiras e Agrometeorologia, Caixa Postal 15100, 91501-970 Porto Alegre, RS, Brasil.

4.
Autor para correspondência: stsmiotto@terra.com.br

\section{Introdução}

A subfamília Caesalpinioideae (Leguminosae) apresenta 2.800 espécies e cerca de 154 gêneros (Raven \& Polhill 1981, Lewis 1987, Lewis \& Polhill 1998). Este grupo pantropical ocorre nos mais variados hábitats, com grande variabilidade de estruturas reprodutivas e vegetativas. A maioria dos gêneros encontram-se nos trópicos, na África, América e sudeste da Ásia, sendo bem representados no Brasil (Ribeiro et al. 1999). Nos 
três Estados da região Sul, pela análise de exsicatas, coletas a campo e revisão de literatura, são encontrados 14 gêneros e cerca de 56 espécies nativas (Bortoluzzi 2004). Alguns gêneros têm seus centros de diversidade e radiação no Brasil, como por exemplo, Chamaecrista Moench (Conceição et al. 2001).

Segundo Polhill et al. (1981) e Burkart (1987) Caesalpinioideae é considerada a subfamília mais primitiva e a menos entendida até o momento (Herendeen 2000). Atualmente, inúmeros estudos biossistemáticos vêm sendo realizados com este grupo, os quais confirmam a hipótese de que a subfamília Caesalpinioideae seja um grupo não natural, compreendendo tribos parafiléticas (Tucker \& Douglas 1994, Käss \& Wink 1996, Tucker 1996, Doyle et al. 1997, 2000, Herendeen 2000, Bruneau et al. 2001, Kajita et al. 2001). Lewis \& Polhill (1998) dividiram-na em quatro tribos: Caesalpinieae, Cassieae, Cercideae e Detarieae, sendo que as duas primeiras são consideradas grupos não naturais, compreendendo clados parafiléticos (Irwin \& Barneby 1981, Tucker \& Douglas 1994, Käss \& Wink 1996, Doyle et al. 1997, 2000, Herendeen 2000, Lewis et al. 2000, Bruneau et al. 2001), estando sujeitas a mudanças no futuro, com novas combinações.

Os táxons desta subfamília estão amplamente distribuídos em diversas formações vegetais ocorrentes na região Sul do Brasil como Floresta Estacional Semidecidual, Floresta Estacional Decidual, Floresta Ombrófila Densa, Floresta Ombrófila Mista, Restingas, Cerrado e Campos Sulinos. Apresentam hábitos variados, desde árvores com mais de $35 \mathrm{~m}$ de altura, como por exemplo, Apuleia leiocarpa (Vogel) J.F. Macbr. e Schizolobyum parahyba (Vell.) S.F. Blake até subarbustos ou ervas, como Chamaecrista rotundifolia (Pers.) Greene, C. repens (Vogel) H.S. Irwin \& Barneby e Senna pilifera (Vogel) H.S. Irwin \& Barneby entre outras (Bortoluzzi 2004).

A subfamília Caesalpinioideae apresenta grande variabilidade intergenérica, interespecífica e intraespecífica de números cromossômicos, sendo citados para espécies brasileiras $2 \mathrm{n}=14,16,22,24,26$, 28, 32, 48 e 52 (Covas 1949, Irwin \& Turner 1960, Bandel 1974, Coleman \& DeMenezes 1980, Gibbs \& Ingram 1982, Alves \& Custódio 1989, Beltrão \& Guerra 1990, Auler et al. 1998, Souza \& Benko-Iseppon, 2004). Goldblatt (1981a) propôs três diferentes números básicos para a subfamília, $\mathrm{x}=7$ e $\mathrm{x}=14$ para as tribos Cassieae, Caesalpinieae e Cercideae e $\mathrm{x}=12$ para as tribos Detarieae e Amherstieae. A poliploidia deve ter sido importante na diversificação inicial do grupo (Bandel 1974, Goldblatt 1981a), sendo que a ocorrência de disploidia é citada como mecanismo de variação do número de cromossomos (Irwin \& Turner 1960, Irwin 1964, Bandel 1974, Goldblatt 1981a, Souza \& BenkoIseppon, 2004).

O número de cromossomos é a característica mais amplamente utilizada em citogenética (Guerra 2000) e, juntamente com outras características citológicas, auxilia no entendimento de variações genéticas envolvidas na evolução de um grupo, como também na delimitação taxonômica de espécies (Pedrosa et al. 1999). Mesmo assim, muitas espécies ainda não tiveram seus números cromossômicos determinados. A subfamília Caesalpinioideae é, juntamente com Mimosoideae, muito pouco estudada citogeneticamente (Guerra 1990), sendo que muitas espécies têm seus números cromossômicos incorretos ou até mesmo desconhecidos, devido ao pequeno número de trabalhos publicados. Portanto, este trabalho teve por objetivos contar o número de cromossomos de 26 espécies incluídas em dez gêneros, pertencentes a três tribos: Cassieae, Caesalpinieae e Cercideae, propor o número básico de cromossomos para os grupos analisados, além de fazer correlações com a taxonomia e filogenia do grupo.

\section{Material e métodos}

Na tabela 1 estão listadas as tribos, locais e datas de coleta e exsicatas depositadas no Herbário ICN, do Departamento de Botânica/UFRGS, Porto Alegre, RS, dos 27 táxons da subfamília Caesalpinioideae ocorrentes na região Sul do Brasil e analisados neste trabalho. O material botânico foi coletado nos estados do Rio Grande do Sul, Santa Catarina e Paraná. As identificações botânicas foram realizadas através da observação dos espécimes em campo, consulta aos especialistas da família Leguminosae e pela análise de exsicatas. Os táxons dos gêneros Cassia L., Chamaecrista Moench e Senna Mill. foram identificados utilizando-se o trabalho de Irwin \& Barneby (1982).

As sementes de todos os acessos (indivíduos) foram escarificadas e postas a germinar em placas de petri por no máximo 72 horas, em germinadores com fotoperíodo controlado e temperaturas entre 25 e $28^{\circ} \mathrm{C}$. As radículas, com cerca de 2 a $3 \mathrm{~cm}$, foram pré-tratadas em solução saturada de paradiclorobenzeno (PDB) por $24 \mathrm{~h} \mathrm{em}$ temperatura de $4{ }^{\circ} \mathrm{C}$. Após, as radículas foram lavadas e fixadas em Carnoy 9:3:1 (etanol: clorofórmio: ácido acético) por $24 \mathrm{~h}$, em temperatura ambiente, sendo, em seguida, estocadas em álcool $70 \% \mathrm{em}$ geladeira.

No preparo das lâminas, foi utilizada a coloração de Feulgen, as radículas foram lavadas em água destilada, hidrolizadas em $\mathrm{HCl} 1 \mathrm{~N} \mathrm{a} 60^{\circ} \mathrm{C}$ por dez minutos, novamente lavadas e colocadas no corante por uma ou, no máximo, duas horas. A ponta da raiz foi esmagada com uma gota de 
Tabela 1. Tribos, locais, datas de coleta e exsicatas de 27 táxons da subfamília Caesalpinioideae (Leguminosae) ocorrentes na região Sul do Brasil.***sem exsicata, **espécies subespontâneas, *planta cultivada.

Table 1. Tribes, places, dates of collection and vouchers of 27 taxa of subfamily Caesalpinioideae (Leguminosae) from the Southern region of Brazil. ***no voucher, **subspontaneous species, *cultivated plant.

Táxons

\section{TRIBOCASSIEAE}

Apuleia leiocarpa (Vogel) Macbr.

Cassia leptophylla Vogel *

Chamaecrista desvauxii (Collad.) Killip

C. flexuosa (L.) Greene

C. nictitans (L.) Moench subsp. patellaria (Collad.) H.S. Irwin \& Barneby

C. nictitans subsp. disadena (Steudel) H.S. Irwin \& Barneby

C. repens (Vogel) H.S. Irwin \& Barneby

C. rotundifolia (Pers.) Greene

C. vestita (Vogel) H.S. Irwin \& Barneby Senna alata (L.) Roxb. **

S. araucarietorum H.S. Irwin \& Barneby
Locais de coleta e/ou exsicatas

BRASIL. Rio Grande do Sul: Turvo, 24-XI-2001, E. Biondo ***

BRASIL. Rio Grande do Sul: Porto Alegre, 20-IV-2002, E. Biondo ***

BRASIL. Paraná: Wensceslau Braz, 31-XII-2001, E. Biondo***

BRASIL. SANTA CATARINA: Laguna, 12-I-2002, R.L.C. Bortoluzzi 1197 (ICN)

BRASIL. Paraná: Capitão Leônidas, 15-IV-2002, E. Biondo 297 (ICN)

BRASIL. Rio Grande Do Sul: Terra de Areia, 16-I-2003, E. Biondo 416 (ICN)

BRASIL. Rio Grande Do Sul: Terra de Areia, 16-I-2003, E. Biondo 417 (ICN)

BRASIL. Rio Grande do Sul: Terra de Areia, 16-I-2003, E. Biondo 419 (ICN)

BRASIL. Rio Grande do Sul: Canoas, 26-I-2001, E. Biondo 179 (ICN)

BRASIL. SAnta CataRina: Pouso Redondo, SC 422, 30-I-2001, E. Biondo 191 (ICN)

BRASIL. Rio GRande do Sul: Terra de Areia, 30-I-2001, S.T.S. Miotto 2071

(ICN)

BRASIL. Rio Grande do Sul: São Borja, BR 472, Km 434, 4-IV-2001, M. Ritter 1356 (ICN)

BRASIL. Rio Grande do Sul: Porto Alegre, 29-V-2001, E. Biondo 199 (ICN)

BRASIL. Rio Grande do Sul: Jaguari, 14-I-2002, E. Biondo 233 (ICN)

BRASIL. Paraná: Arapoti, PR 151, 20-III-2002, R.L.C. Bortoluzzi \& E. Biondo $1163(\mathrm{ICN})$

BRASIL. Paraná: Carambeí, PR 151,19-III-2002, E. Biondo 258 (ICN)

BRASIL. Paraná: Capitão Leônidas, 15-IV-2002, E. Biondo 296 (ICN)

BRASIL. PARANÁ: Xambrê, 16-IV-2002, E. Biondo 306 (ICN)

BRASIL. Rio Grande do Sul: Santo Antônio das Missões, BR 285, Km 607, 25-IV-2002, E. Biondo 363 (ICN)

BRASIL. Rio Grande do Sul: São Miguel das Missões, 25-IV-2002, E. Biondo $355(\mathrm{ICN})$

BRASIL. Santa Catarina: Camboriú, BR 101, Km 130, 10-III-2001, E. Biondo 198 (ICN)

BRASIL. Santa Catarina: Massiambu, BR 101, Km 233, 20-X-2001, E. Biondo 208 (ICN)

BRASIL. SANTA CATARINA: Tubarão, BR 101, Km 336, 19-X-2001, E. Biondo 206 (ICN)

BRASIL. Rio Grande do Sul: Santa Maria, 2-I-2001, E. Biondo ***

BRASIL. Rio Grande do Sul: Cachoeira do Sul, BR 290, Km 311, 11-I-2002, S.T.S. Miotto 2000 (ICN)

BRASIL. Rio Grande do Sul: Cachoeira do Sul, BR 290, Km 311, 11-I-2002, E. Biondo 223 (ICN)

BRASIL. Rio GRANDE Do Sul: Santana do Livramento, RS 158, Km 520, 12-I-2002, S.T.S. Miotto 2011 (ICN)

BRASIL. ParanÁ: Jaguariaíva, PR 151, Km 234, 19-III-2002, E. Biondo 262 (ICN)

BRASIL. Paraná: Castro, PR 151, Km 272, 18-III-2003, E. Biondo 435 (ICN)

BRASIL. PARANÁ: Marmelândia, 15-IV-2002, E. Biondo 294 (ICN)

BRASIL. Santa Catarina: Criciúma, BR 101, Km 395, 22-III-2002, E. Biondo $280(\mathrm{ICN})$ 
BRASIL. SANTA CATARINA: Criciúma, BR 101, Km 395, 22-III-2002, E. Biondo $281(\mathrm{ICN})$

BRASIL. Santa Catarina: Concórdia, BR 153, Km 86, 18-III-2002, R.L.C. Bortoluzzi \& E. Biondo 1152 (ICN)

BRASIL. Santa Catarina: Concórdia, BR 153, Km 86, 18-III-2002, R.L.C. Bortoluzzi \& E. Biondo 1151 (ICN)

S. corymbosa (Lam.) H.S. Irwin \& Barneby BRASIL. Rio Grande do Sul: Santa Cruz do Sul, 18-VIII-2003, E. Biondo 444 (ICN)

S. hilariana (Benth.) H.S. Irwin \& Barneby BRASIL. Rio Grande Do Sul: Canoas, 29-XII-2000, A.S. Flores \& R.S. Rodrigues $402(\mathrm{ICN}) *$

BRASIL. Rio Grande do Sul: Entre Ijuís, Sítio Arqueológico de São João Batista - Missões, 25-IV-2002, E. Biondo 353 (ICN)

S. hirsuta (L.) H.S. Irwin \& Barneby

BRASIL. Rio Grande do Sul: Veranópolis, Km 5, 21-V-2000, M. Ritter 1187 (ICN)

BRASIL. Rio Grande do Sul: Getúlio Vargas, RS 135, Km 24, 18-III-2002, E. Biondo 245 (ICN)

BRASIL. SANTA Catarina: Seara, 14-IV-2004, E. Biondo 284 (ICN)

BRASIL. SANTA Catarina: Chapecó, BR 480, 14-IV-2004, E. Biondo 286 (ICN)

BRASIL. Rio GRANDE do Sul: Ijuí, RS 342, 25-IV-2002, E. Biondo 336 (ICN)

S. macranthera (DC. ex Collad.) H.S. $\quad$ BRASIL. PARANÁ: Assis Chateaubriant, 26-IV-2002, R.L.C. Bortoluzzi 1244 Irwin \& Barneby (ICN)

S. multijuga (L.C. Rich.) H.S. Irwin \& Barneby BRASIL. PARANÁ: Cascavel, BR 277, 15-IV-2002, R.L.C. Bortoluzzi \& E. Biondo $1238(\mathrm{ICN})$

S. neglecta (Vogel) H.S. Irwin \& Barneby BRASIL. SANTA CATARINA: Vidal Ramos, 21-X-2001, E. Biondo 215 (ICN) BRASIL. SANTA CATARINA: Rancho Queimado, 21-X-2001, E. Biondo 207 (ICN) BRASIL. PARANá: Doutor Ulisses, 20-III-2002, E. Biondo 270 (ICN)

BRASIL. PARANá: Mallet, PR 153, acesso secundário, 19-III-2002, E. Biondo $248(\mathrm{ICN})$

S. oblongifolia (Vogel) H.S. Irwin \& Barneby

BRASIL. Rio Grande do Sul: Bom Jesus, RS 110, Km 4, 15-I-2000, S.T.S. Miotto $1934(\mathrm{ICN})$

BRASIL. Rio Grande do Sul: Bom Jesus, RS 110, Km 4, 15-I-2000, S.T.S. Miotto $1933(\mathrm{ICN})$

BRASIL. Rio Grande do Sul: São Marcos, 27-I-2001, S.T.S. Miotto 2043 (ICN)

S. obtusifolia (L.) H.S. Irwin \& Barneby **

BRASIL. Paraná: Toledo, PR 486, 15-IV-2002, E. Biondo 298 (ICN)

BRASIL. PARANÁ: Juranda, Ribeirão Verde, 16-IV-2002, R.L.C. Bortoluzzi \& E. Biondo 1247 (ICN)

BRASIL. Rio Grande do Sul: Entre Ijuís, BR 285, Km 607, 25-IV-2002, E. Biondo 346 (ICN)

S. occidentalis (L.) Link **

BRASIL. Rio Grande do Sul: Itaqui, BR 472, Km 478, 4-IV-2001, M. Ritter 1360 (ICN)

BRASIL. Rio GRANDE do Sul: São Borja, 2-IV-2001, M. Ritter 1344 (ICN)

BRASIL. Rio Grande do Sul: Santo Antônio das Missões, BR 285, Km 607 , 25-IV-2002, E. Biondo 361 (ICN)

BRASIL. Rio Grande do Sul: Entre Ijuís, 25-IV-2002, E. Biondo 350 (ICN)

S. pilifera (Vogel) H.S. Irwin \& Barneby

BRASIL. Rio GrandE Do Sul: São Borja, BR 287, 13-I-2002, S.T.S. Miotto 2032 (ICN)

BRASIL. Rio Grande do Sul: São Borja, BR 387, 13-I-2002, E. Biondo 230 (ICN)

BRASIL. Rio Grande do Sul: São Borja, BR 387, 13-I-2002, E. Biondo 231 (ICN) 


\section{TRIBOCAESALPINIEAE}

Gleditsia amorphoides (Griseb.) Taub.

Parkinsonia aculeata L.

Peltophorum dubium (Spreng.) Taub.

Pomaria stipularis (Vogel) B.B. Simpson \& G.P. Lewis

Schizolobium parahyba (Vell.) S.F. Blake

TRIBOCERCIDAE

Bauhinia forficata Link
BRASIL. Rio Grande do Sul: Dilermando de Aguiar, 26-VI-2001, E. Biondo*** BRASIL. Rio Grande do Sul: Porto Alegre, 12-I-2001, E. Biondo 88* (ICN)

BRASIL. Rio Grande do Sul: Porto Alegre, 12-I-2001, E. Biondo 89* (ICN)

BRASIL. Rio Grande do Sul: Porto Alegre, 25-V-2001, E. Biondo ***

BRASIL. Santa Catarina: Mondaí, 23-V-2001, R.L.C. Bortoluzzi et al. 984 (ICN)

BRASIL. Paraná: Maringá, 17-IV-2002, E. Biondo 307 (ICN)

BRASIL. PARANÁ: Jaguariaíva, PR 151, 13-XII-1998, E. Biondo ***

BRASIL. Rio Grande do Sul: Porto Alegre, 23-XI-2000, E. Biondo ***, * BRASIL. Santa Catarina: Brusque, 20-X-2001, R.L.C. Bortoluzzi \& E. Biondo $1021(\mathrm{ICN})$

BRASIL. Santa CATARINA: Campo Erê, 24-V-2001, R.L.C. Bortoluzzi et al. 989 (ICN)

BRASIL. PARANÁ: Juranda, 16-IV-2002, E. Biondo 303 (ICN)

BRASIL. Paraná: Cascavel, 15-IV-2002, R.L.C. Bortoluzzi \& E. Biondo 1239 (ICN)

BRASIL. Paraná: Realeza, 15-IV-2002, E. Biondo 295 (ICN) carmim propiônico $2 \%$. Foram contadas, um mínimo de três células e máximo de 281 células por espécie (tabela 2). As análises foram realizadas nas células cujos cromossomos apresentavam-se espalhados e, quando possível, bem condensados, em microscópio ótico com aumento de 1.000 vezes. As melhores metáfases foram fotografadas.

\section{Resultados}

Foram estudados 74 acessos de 27 táxons incluídos em 10 gêneros (tabelas 1, 2). Destes, sete espécies tiveram seus números cromossômicos contados pela primeira vez, são elas: Cassia leptophylla Vogel, Senna araucarietorum H.S. Irwin \& Barneby (figura 2), S. hilariana (Benth.) H.S. Irwin \& Barneby (figura 4), S. neglecta (Vogel) H.S. Irwin \& Barneby (figura 3), S. oblongifolia (Vogel) H.S. Irwin \& Barneby (figura 5), Chamaecrista repens (tribo Cassieae) e Pomaria stipularis (Vogel) B.B. Simpson \& G.P. Lewis (figura 6) (tribo Caesalpinieae) (tabela 2).

Para a tribo Cassieae foram contados $2 \mathrm{n}=28$ cromossomos em Apuleia leiocarpa (um acesso) (figura 1), Cassia leptophylla (um acesso), S. alata (L.) Roxby (um acesso), S. araucarietorum (quatro acessos) (figura 2), S. corymbosa (um acesso), S. hilariana (dois acessos) (figura 4), S. hirsuta (L.)
H.S. Irwin \& Barneby (cinco acessos), S. neglecta (quatro acessos) (figura 3), S. occidentalis (L.) Link (quatro acessos), S. oblongifolia (três acessos) (figura $5) ; 2 \mathrm{n}=26 \mathrm{em}$ S. macranthera (DC. ex Collad.) H.S. Irwin \& Barneby (um acesso) e em S. obtusifolia (três acessos); 2n = 24 em $S$. multijuga (L.C. Rich) H.S. Irwin \& Barneby (um acesso) e $2 \mathrm{n}=22 \mathrm{em}$ S. pilifera (três acessos). Chamaecrista nictitans (L.) Moench subsp. patellaria (Collad.) H.S. Irwin \& Barneby (12 acessos) e $C$. nictitans subsp. disadena (Steudel) H.S. Irwin \& Barneby (três acessos) possuem $2 \mathrm{n}=32$ cromossomos; para C. repens (quatro acessos), C. flexuosa (L.) Greene (três acessos), C. rotundifolia (Pers.) Greene (um acesso) e C. vestita (Vogel) H.S. Irwin \& Barneby (um acesso) foram contados $2 \mathrm{n}=16$ cromossomos e $C$. desvauxii (Collad.) Killip (três acessos) possui $2 \mathrm{n}=14$ cromossomos (tabela 2 ).

Cinco espécies da tribo Caesalpinieae foram analisadas quanto ao número de cromossomos, sendo que Gleditsia amorphoides (Griseb.) Taub. (um acesso) e Parkinsonia aculeata L. (dois acessos) possuem 2n = 28; Peltophorum dubium (Spreng.) Taub. (três acessos) possui $2 \mathrm{n}=26$ e Schizolobium parahyba (dois acessos) e Pomaria stipularis (um acesso) (figura 6) possuem $2 \mathrm{n}=24$ cromossomos (tabela 2 ).

Para a tribo Cercideae, apenas uma espécie foi 
Tabela 2. Tribos, números de acessos, números de células analisadas, número cromossômico de 27 táxons pertencentes a dez gêneros da subfamília Caesalpinioideae (Leguminosae) ocorrentes na região Sul do Brasil. $\mathrm{N}$ de $\mathrm{AC}=$ número de acessos, entre parênteses número de células analisadas; $\mathrm{n}=$ número haplóide de cromossomos; $2 \mathrm{n}=$ número diplóide de cromossomos; *contagens inéditas.

Table 2. Tribes, number of accessions, number of cells analyzed and chromosome numbers of 27 taxa of ten genus of subfamily Caesalpinioideae (Leguminosae) from the Southern region of Brazil. N de AC = number of accessions analysed, between brackets number of cells analysed; $\mathrm{n}=$ haploid chromosome number; $2 \mathrm{n}=$ diploid chromosome number; $*$ original counts.

\begin{tabular}{|c|c|c|c|c|c|}
\hline \multirow[t]{2}{*}{ Táxons } & \multirow[t]{2}{*}{$\mathrm{N}$ de $\mathrm{AC}$} & \multirow[t]{2}{*}{$2 n$} & \multicolumn{3}{|r|}{ Determinações prévias } \\
\hline & & & $\mathrm{n}$ & $2 n$ & Referências \\
\hline \multicolumn{6}{|l|}{ TRIBOCASSIEAE } \\
\hline Apuleia leiocarpa & $1(21)$ & 28 & - & 26,28 & Goldblatt 1981b, Auler 1997, Auler et al. 1998 \\
\hline Cassia leptophylla* & $1(35)$ & 28 & - & - & - \\
\hline Chamaecrista desvauxii & $3(75)$ & 14 & 7 & & $\begin{array}{l}\text { Irwin \& Turner } 1960 \text {, Federov 1969, Ormond } \\
\text { et al. } 1977 \text {, Coleman \& De Menezes } 1980\end{array}$ \\
\hline Chamaecrista flexuosa & $3(22)$ & 16 & 8 & 16 & $\begin{array}{l}\text { Irwin \& Turner 1960, Federov 1969, Coleman \& } \\
\text { De Menezes 1980, Goldblatt \& Johnson } 2000\end{array}$ \\
\hline $\begin{array}{l}\text { Chamaecrista nictitans subsp. } \\
\text { patellaria }\end{array}$ & $12(281)$ & 32 & 8 & $16,32,48$ & $\begin{array}{l}\text { Irwin \& Turner } 1960, \text { Coleman \& De Menezes } \\
1980 \\
\text { Irwin \& Turner } 1960 \text {, Souza } 2000, \text { Souza et al. } \\
2000\end{array}$ \\
\hline $\begin{array}{l}\text { Chamaecrista nictitans subsp. } \\
\text { disadena }\end{array}$ & $3(79)$ & 32 & 16 & 32 & Irwin \& Turner 1960, Federov 1969 \\
\hline Chamaecrista repens $*$ & $4(70)$ & 16 & - & - & - \\
\hline Chamaecrista rotundifolia & $1(18)$ & 16 & 8 & $14,16,32$ & $\begin{array}{l}\text { Miége 1960, Irwin \& Turner 1960, Federov 1969, } \\
\text { Coleman \& De Menezes 1980, Goldblatt \& } \\
\text { Johnson } 1994\end{array}$ \\
\hline Chamaecrista vestita & $1(38)$ & 16 & 8 & & Irwin \& Turner 1960, Federov 1969 \\
\hline Senna alata & $1(11)$ & 28 & 14 & 24,28 & $\begin{array}{l}\text { Irwin \& Turner 1960, Federov 1969, Gill \& } \\
\text { Husaini 1985, Godblatt 1988, Alves \& Custódio } \\
\text { 1989, Goldblatt \& Johnson 1990, 1991, 1996, } 2000 \text {, } \\
\text { Souza et al. 2000, Souza \& Benko-Iseppon } 2004\end{array}$ \\
\hline Senna araucarietorum * & $4(47)$ & 28 & - & - & - \\
\hline Senna corymbosa & $1(09)$ & 28 & 14 & 28 & Irwin \& Turner 1960, Federov 1969 \\
\hline Senna hilariana * & $2(26)$ & 28 & - & - & - \\
\hline Senna hirsuta & $5(130)$ & 28 & $\begin{array}{c}8+1 b, 14 \\
16+1 B\end{array}$ & 28,56 & $\begin{array}{l}\text { Frahm-Leliveld 1957, Irwin \& Turner 1960, } \\
\text { Federov 1969, Gill \& Husaini 1985, Goldblatt } \\
\text { 1988, Goldblatt \& Johnson, } 1996\end{array}$ \\
\hline Senna macranthera & $1(03)$ & 26 & & 24,26 & Irwin \& Turner 1960, Federov 1969, Bandel 1974 \\
\hline Senna multijuga & $1(08)$ & 24 & 12,16 & 16,24 & $\begin{array}{l}\text { Irwin \& Turner 1960, Federov 1969, Bandel 1974, } \\
\text { Coleman \& De Menezes 1980, Gill \& Husaini } \\
\text { 1985, Goldblatt } 1988\end{array}$ \\
\hline Senna neglecta * & $4(43)$ & 28 & - & - & - \\
\hline Senna oblongifolia * & $3(38)$ & 28 & - & - & - \\
\hline Senna obtusifolia & $3(30)$ & 26 & 13,14 & $\begin{array}{l}26,28 \\
52,56\end{array}$ & $\begin{array}{l}\text { Irwin \& Turner 1960, Federov 1969, Gill \& } \\
\text { Husaini 1985, Goldblatt 1988, Alves \& Custódio } \\
\text { 1989, Goldblatt \& Johnson 1990, 1996, 2000, } \\
\text { Souza et al. 2000, Souza \& Benko-Iseppon } 2004\end{array}$ \\
\hline Senna occidentalis & $4(82)$ & 28 & 13,14 & $\begin{array}{l}24,26 \\
28,56\end{array}$ & $\begin{array}{l}\text { Burkart 1952, Turner 1956, Irwin \& Turner 1960, } \\
\text { Federov 1969, Coleman \& De Menezes 1980, } \\
\text { Gill \& Husaini 1985, Goldblatt 1985, 1988, Ohri } \\
\text { et al. 1986, Jahan et al. 1994, Goldblatt \& } \\
\text { Johnson 1990, 1991, 1994, 1996, 1998, Ghareeb } \\
\text { et al. } 1999\end{array}$ \\
\hline
\end{tabular}


continuação

\begin{tabular}{|c|c|c|c|c|c|}
\hline \multirow[t]{2}{*}{ Táxons } & \multirow[t]{2}{*}{$\mathrm{N}$ de $\mathrm{AC}$} & \multirow[t]{2}{*}{$2 n$} & \multicolumn{3}{|r|}{ Determinações prévias } \\
\hline & & & $\mathrm{n}$ & $2 n$ & Referências \\
\hline Senna pilifera & $3(64)$ & 22 & 11 & & $\begin{array}{l}\text { Irwin \& Turner 1960, Federov 1969, Coleman \& } \\
\text { De Menezes } 1980\end{array}$ \\
\hline \multicolumn{6}{|l|}{ TRIBOCAESALPINIEAE } \\
\hline Gleditsia amorphoides & $1(03)$ & 28 & & 28 & Castronovo 1945, Federov 1969 \\
\hline Parkinsonia aculeata & $2(55)$ & 28 & 9 & 28 & $\begin{array}{l}\text { Federov 1969, Bandel 1974, Gill \& Husaini 1985, } \\
\text { Goldblatt 1988, Kumari \& Bir 1989, Jahan et al. } \\
\text { 1994, Goldblatt \& Johnson 1991, 1994, } 1998\end{array}$ \\
\hline Peltophorum dubium & $3(51)$ & 26 & & 26,28 & $\begin{array}{l}\text { Gibbs \& Ingram 1982, Goldblatt 1985, Kumari \& } \\
\text { Bir } 1989\end{array}$ \\
\hline Pomaria stipularis $*$ & $1(48)$ & 24 & - & - & - \\
\hline Schizolobium parahyba & $2(13)$ & 24 & & 24 & Gibbs \& Ingram 1982, Goldblatt 1985 \\
\hline \multicolumn{6}{|l|}{ TRIBOCERCIDEAE } \\
\hline Bauhinia forficata & $4(45)$ & 28 & - & 28 & $\begin{array}{l}\text { Gill \& Husaini 1985, Goldblatt 1988, Goldblatt \& } \\
\text { Johnson } 1990\end{array}$ \\
\hline
\end{tabular}

analisada, Bauhinia forficata Link (três acessos) a qual apresentou $2 \mathrm{n}=28$ cromossomos (tabela 2 ).

\section{Discussão}

Foram estudadas 26 espécies (tabelas 1, 2), representando cerca de $46 \%$ do total de espécies pertencentes à subfamília Caesalpinioideae ocorrentes na região Sul. As determinações do número de cromossomos em sete espécies foram consideradas inéditas, não tendo sido encontradas em revisão de literatura (Federov 1969, Goldblatt 1981b, 1985, 1988, Goldblatt \& Johnson 1990, 1991, 1994, 1996, 1998, 2000) (tabela 2). Os cromossomos são pequenos com tamanho em torno de 1,5 a $2,5 \mathrm{~mm}$, sendo que os maiores cromossomos são observados em Pomaria stipularis com cerca de $2,9 \mathrm{~mm}$.

Tribo Cassieae - O número de cromossomos observado em Apuleia leiocarpa, concorda com o citado por Goldblatt (1981b). Já, Auler et al. (1998) encontraram $2 n=26$ cromossomos em indivíduos de uma população de Floresta Estacional Semidecidual. Goldblatt (1981a) citou $2 n=24$ e 28 para algumas espécies da subtribo Dialiineae à qual pertence Apuleia leiocarpa. Neste trabalho, apenas um acesso foi analisado, entretanto, como pode ser observado na figura 1 , não existem dúvidas quanto ao número cromossômico. A análise de indivíduos de diferentes populações de uma mesma espécie é muito importante, já que variações significativas de números cromossômicos entre populações são encontradas em literatura (Forni-Martins \& Martins 2000).

Cassia leptophylla possui $2 \mathrm{n}=28$ cromossomos, sendo esta a primeira citação para a espécie (tabela 2). O número de cromossomos para algumas espécies de Cassia (s.str.), como por exemplo, C. grandis L. e C. javanica L.é 2n = 28 (Goldblatt 1985, 1988, Goldblatt \& Johnson 1996), tendo sido citado também $2 \mathrm{n}=24$ cromossomos para C. fistula L. (Goldblatt 1985, 1988, Goldblatt \& Johnson 1990, 1991, 1994, 1996) e, para C. fastuosa Willd. ex Vogel, $2 \mathrm{n}=26$ (Souza \& BenkoIseppon 2004). Estas variações são provavelmente devidas à disploidia, mecanismo de variação do número cromossômico, muito comum nas espécies da tribo Cassieae (Irwin \& Turner 1960, Irwin 1964, Bandel 1974, Goldblatt 1981a, Souza \& Benko-Iseppon 2004). Contudo, a hipótese de eventuais contagens errôneas, muitas vezes por determinações taxonômicas incorretas, também não pode ser descartada, já que estas não são raras em literatura (Guerra 1990).

Senna hirsuta e S. occidentalis com $2 \mathrm{n}=28$ cromossomos (tabela 2) tiveram seus números cromossômicos determinados por diversos autores (tabela 2). Destas, somente S. hirsuta é nativa na região Sul do Brasil, sendo citada para esta espécie a ocorrência de cromossomos B (Gill \& Husaini 1985, Goldblatt 1988, Goldblatt \& Johnson 1996), os quais não foram observados nos indivíduos desta espécie analisados neste trabalho. Para S. occidentalis, subespontânea na região Sul do Brasil, quatro diferentes 

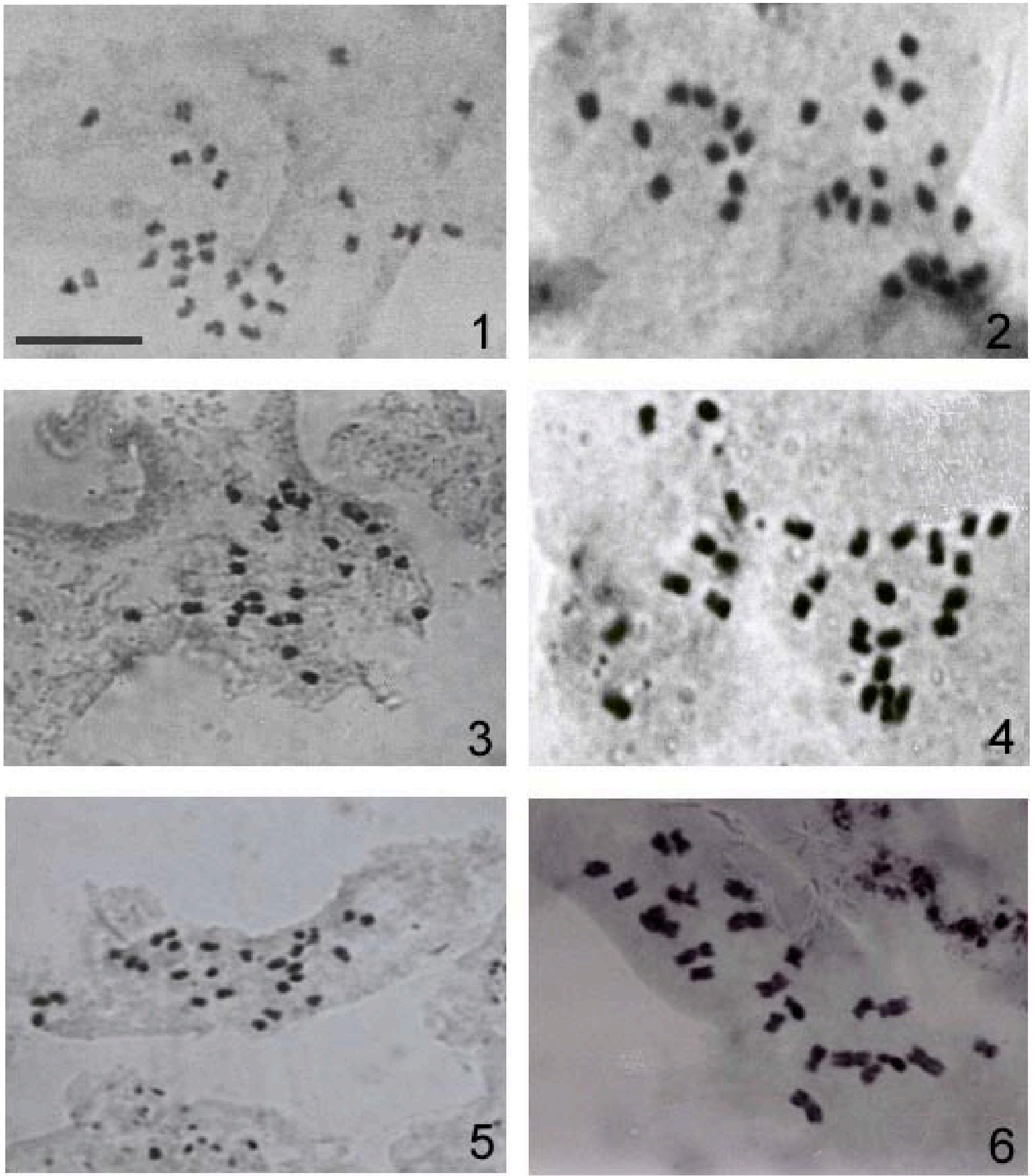

Figuras 1-6. Metáfases mitóticas em espécies da subfamília Caesalpinioideae. 1. Apuleia leiocarpa, $2 \mathrm{n}=28$ cromossomos. 2. Senna araucarietorum, $2 \mathrm{n}=28$. 3. S. neglecta, $2 \mathrm{n}=28$. 4. Senna hilariana, $2 \mathrm{n}=28$. 5. S. oblongifolia, $2 \mathrm{n}=28$. 6. Pomaria stipularis, $2 \mathrm{n}=24$. Barra $=10 \mu \mathrm{m}$.

Figures 1-6. Mitotic metaphases in species of subfamily Caesalpinioideae. 1. Apuleia leiocarpa, $2 \mathrm{n}=28$ chromosomes. 2. Senna araucarietorum, $2 \mathrm{n}=28$. 3. S. neglecta, $2 \mathrm{n}=28$. 4. Senna hilariana, $2 \mathrm{n}=28$. 5. S. oblongifolia, $2 \mathrm{n}=28$. 6. Pomaria stipularis, $2 \mathrm{n}=24$. $\mathrm{Bar}=10 \mu \mathrm{m}$. 
números cromossômicos são citados $2 \mathrm{n}=24,26,28 \mathrm{e}$ 56 (tabela 2), sendo que, freqüentemente, são citados $\mathrm{n}=13$ e 14 cromossomos para esta espécie (Burkart 1952, Coleman \& DeMenezes 1980). Ambas estão amplamente distribuídas pelo mundo (Irwin \& Barneby 1982), o que poderia justificar o grande número de análises realizadas com estas duas espécies em comparação com as demais espécies da subfamília. Devido à grande variabilidade intraespecífica constatada para estas espécies em diversos trabalhos (tabela 2), é possível que contagens incorretas também tenham sido realizadas.

Número cromossômico $2 \mathrm{n}=26$ foi observado em S. macranthera (tabela 2). Bandel (1974) citou $2 n=24$ para esta mesma espécie coletada na região Sudeste, sendo constatada, portanto, uma variação no número de cromossomos entre populações de uma mesma espécie com diferentes distribuições geográficas. No presente trabalho, um pequeno número de células foram analisadas (tabela 2) e, embora possam ser contados 26 cromossomos, sugere-se uma análise mais abrangente.

Nos acessos de $S$. obtusifolia foram contados $2 n=26$ cromossomos (tabela 2). Para populações amazônicas desta espécie, Souza \& Benko-Iseppon (2004) citaram $2 \mathrm{n}=26$. O mesmo número cromossômico foi citado por Alves \& Custódio (1989) para indivíduos ocorrentes no estado do Ceará e $\mathrm{n}=13$ foi citado por Coleman \& De Menezes (1980) para espécimes do estado de São Paulo. Diferentes números cromossômicos para a espécie foram encontrados em literatura, sendo citados $2 \mathrm{n}=26,28$, 52 e 56 cromossomos (tabela 2). A ampla variabilidade intraespecífica nesta espécie foi observada em populações ocorrentes em regiões paleotropicais, constatando-se um número constante de $2 \mathrm{n}=26$ e $\mathrm{n}=13$ cromossomos em indivíduos pertencentes à essa espécie com ampla distribuição no território brasileiro.

Senna multijuga apresentou $2 \mathrm{n}=24$ cromossomos (tabela 2), o mesmo número cromossômico citado por Bandel (1974) para indivíduos ocorrentes nas regiões Centro Oeste e Sudeste. Porém, n = 16 (Goldblatt 1988) e $n=12$ (Irwin \& Turner 1960, Federov 1969, Coleman \& De Menezes 1980) também foram citados. É possível que esteja sendo constatada variabilidade intraespecífica de número cromossômico, com variação relacionada a diferentes regiões de distribuição geográfica da espécie, porém, como mencionado anteriormente, não se pode descartar a hipótese de erros nas contagens, sendo necessárias mais análises.
S. pilifera possui $2 \mathrm{n}=22$ cromossomos (tabela 2 ), o mesmo número cromossômico citado em outros trabalhos realizados com a espécie (Irwin \& Turner 1960, Federov 1969, Coleman \& De Menezes 1980). Esta é a única espécie dentro do gênero Senna, analisada até o momento, que apresentou $2 \mathrm{n}=22$ cromossomos, podendo ser o representante final da série displóide observada neste gênero (Biondo 2004).

No gênero Senna pode-se observar predomínio do número cromossômico $2 \mathrm{n}=28$, encontrado em mais de $65 \%$ das espécies aqui analisadas (tabela 2) e da grande maioria das espécies de Senna estudadas citogenéticamente (Goldblatt 1981a). Devido a isto, reforça-se a hipótese, já proposta em diversos trabalhos, de que $\mathrm{x}=14$ seja o número básico deste gênero (Irwin \& Turner 1960, Goldblatt 1981a). Os demais números cromossômicos $2 n=26,24$ e 22 provavelmente evoluíram por disploidia, ocorrida durante a diversificação do grupo. Entretanto, considerando que este gênero é composto de um grande número de espécies, aproximadamente 260 (Irwin \& Barneby 1982), ainda é necessária prudência nas considerações evolutivas.

Uma série de trabalhos com contagens cromossômicas, para espécies do gênero Chamaecrista foram encontrados em literatura, com predomínio de $2 \mathrm{n}$ $=16$ e $\mathrm{n}=8$ (tabela 2 ). O número básico $\mathrm{x}=8$ foi citado para este gênero, com $\mathrm{x}=7$ na seção Xerocalyx onde inclui-se C. desvauxii (Goldblatt 1981a, Irwin 1964, Irwin \& Barneby 1982). Para os táxons aqui analisados o número básico é x $=8$ e x $=7$, para $C$. desvauxii.

No caso de $C$. nictitans subsp. patellaria observa-se a ocorrência de dois níveis de ploidia, considerando-se $\mathrm{x}=8$ como número básico principal. O nível tetraplóide com $2 \mathrm{n}=4 \mathrm{x}=32$ cromossomos para indivíduos ocorrentes nas regiões Centro Oeste, Sudeste e Sul (Irwin \& Turner 1960, Coleman \& De Menezes 1980, neste trabalho) e o hexaplóide com $2 \mathrm{n}=6 \mathrm{x}=48$ para os indivíduos ocorrentes na Amazônia Oriental (Souza \& Benko-Iseppon 2004). Estes diferentes níveis de ploidia podem indicar a ocorrência de citótipos diferenciados dentro do táxon, tendo em vista as diferentes condições ambientais às quais estão expostos os indivíduos. Neste caso, é muito importante que sejam ampliadas as contagens e coletas para maior entendimento desta questão. Como sugerido por Souza \& Benko-Iseppon (2004) a poliploidia parece ser um evento comum neste gênero.

$\mathrm{Na}$ maioria das espécies analisadas da tribo Cassieae (figuras 2-6, 8), os cromossomos são pequenos, com cerca de $2,5 \mathrm{~mm}$ e predomínio de cromossomos metacêntricos. Souza \& Benko-Iseppon 
(2004) observaram tamanho de cromossomos variando de 0,62 até 2,5 $\mathrm{mm}$, para espécies de Senna e Chamaecrista. Esta tribo apresenta espécies tetraplóides, cujo número básico é $\mathrm{x}=14$, derivado por poliploidia de $\mathrm{x}=7$, citado para o gênero Cercis L., um dos mais basais dentro da família Leguminosae (Polhill et al. 1981). O gênero Chamaecrista é uma exceção, apresentando $\mathrm{x}=8$, sendo que a seção mais especializada, Xerocalyx (Irwin 1964, Irwin \& Barneby 1982), possui $x=7$ que provavelmente derivou por disploidia (Goldblatt 1981a).

Tribo Caesalpinieae - Os números cromossômicos aqui observados concordam com análises prévias (Castronovo 1945, Federov 1969, Bandel 1974, Gibbs \& Ingram 1982, Gill \& Husaini, 1985, Goldblatt 1985, 1988, Kumari \& Bir 1989, Jahan et al. 1994, Goldblatt \& Johnson 1990, 1991, 1994, 1998). Pomaria stipularis, $\operatorname{com} 2 \mathrm{n}=24$ cromossomos, tem seu número de cromossomos determinado pela primeira vez. Este gênero foi reestabelecido recentemente, desmembrado de Caesalpinia L. e Hoffmanseggia Cav., sendo composto por sete espécies. Destas, quatro ocorrem na América do Sul (Simpson \& Lewis 2003). Goldblatt (1981a) sugere que $\mathrm{x}=14$ seja o número básico para a tribo, com $x=13$ e 12 também derivados por disploidia.

Tribo Cercideae - Bauhinia forficata apresentou $2 \mathrm{n}=28$ cromossomos (tabela 2 ), o mesmo número cromossômico observado em outros estudos (Gill \& Husaini 1985, Goldblatt 1988, Goldblatt \& Johnson 1990). Este é o único gênero da tribo Cercideae que ocorre na região Sul do Brasil, sendo considerado de taxonomia complexa (Bortoluzzi 2004). O número básico parece ser $\mathrm{x}=14$. Goldblatt (1981a) propôs $\mathrm{x}=7$ ou $\mathrm{x}=14$ para esta tribo, o mesmo das tribos Cassieae e Caesalpinieae.

Evolução do número básico - As espécies pertencentes às tribos Cassieae e Caesalpinieae possuem número básico cromossômico $\mathrm{x}=14,13$ e 12, confirmando o sugerido por Irwin e Barneby (1982), sendo que Chamaecrista é a exceção com x $=8$ e 7. Bauhinia forficata, pertencente à tribo Cercideae, possui número básico $\mathrm{x}=14$ cromossomos, o qual já foi observado por Goldblatt (1981a).

Com base nestes resultados pode-se levantar a hipótese de que o gênero Cercis com $\mathrm{x}=7$, seria o ancestral comum e mais primitivo, que teria dado origem a todos os demais grupos de Caesalpinioideae, com $\mathrm{x}=14$, surgidos através de poliploidia, a qual teria tido papel fundamental na evolução inicial do grupo, como sugerido por Goldblatt (1981a). A partir daí teria ocorrido uma série de reduções displóides, com alguns representantes ainda existentes como Senna obtusifolia com x $=13, S$. multijuga com x $=12$, S. pilifera com $\mathrm{x}=11$ e Chamaecrista rotundifolia com $\mathrm{x}=8$. No outro extremo, estariam as espécies da seção Xerocalyx, neste trabalho representada por C. desvauxii com $\mathrm{x}=7$, que seriam os representantes mais derivados dentro da subfamília Caesalpinioideae, como sugerido por Irwin (1964) e os representantes finais da série de reduções displóides na subfamília.

Tratando-se especificamente dos gêneros Cassia (s.str.), Senna e Chamaecrista, incluídos na subtribo Cassiinae (Cassieae) e desmembrados por Irwin \& Barneby (1981, 1982) de Cassia (s.l.), foram constatadas diferenças evidentes no número de cromossomos (tabela 2). As espécies de Cassia e Senna analisadas apresentaram número cromossômico $2 n=22$, 24, 26 e 28, enquanto as de Chamaecrista apresentaram $2 \mathrm{n}=14,16$ e 32 (tabela 2). Diferenças citogenéticas entre estes três gêneros, também foram observadas por Souza \& Benko-Iseppon (2004), os quais constataram diferenças quanto ao número e tamanho dos cromossomos, além de padrão de condensação diferenciado de cromossomos profásicos, com Senna e Chamaecrista separando-se de Cassia.

Alguns trabalhos recentes, com análises moleculares, sugerem que o gênero Chamaecrista, embora apresente características que o agrupem a Senna e Cassia, dentro da subtribo Cassiinae, não seja um grupo irmão. Características como composição química específica e nódulos radiculares separam Chamaecrista, nas árvores geradas a partir de análises moleculares, dos outros dois gêneros, reforçando a idéia de segregação destes três gêneros, com futura criação de outra subtribo ou até uma nova tribo (Polhill 1994, Doyle et al. 1997, Lewis \& Polhill 1998, Bruneau et al. 2001).

Portanto, pelo que foi discutido, além dos caracteres morfológicos, que foram a base para a separação dos três gêneros, os caracteres citogenéticos também corroboraram esta segregação. Além disto, embora seja prematura, principalmente devido ao pequeno volume de informações, mas com base nas diferenças observadas em diferentes trabalhos, sugere-se a separação do gênero Chamaecrista dos demais gêneros da subtribo Cassiinae, como proposto em outros trabalhos. Contudo, é importante ressaltar que muitas espécies ainda não foram analisadas e nem revisadas taxonômicamente, sendo que ainda são necessários muitos estudos biossistemáticos para que isto seja confirmado. 
Agradecimentos - À Capes pela bolsa de pesquisa concedida à primeira autora e ao $\mathrm{CNPq}$ pelo auxílio financeiro e pelas Bolsas de Produtividade à segunda e à terceira autoras. A Roseli Lopes da Costa Bortoluzzi e Rafael Trevisan pela montagem da prancha fotográfica.

\section{Referências bibliográficas}

ALVES, M.A.O. \& CUSTÓDIO, A.V.C. 1989. Citogenética de Leguminosas coletadas no estado do Ceará. Revista Brasileira de Genética 12:81-92.

AULER, N.M.F. 1997. Estudo citogenético e anatomia da madeira de Apuleia leiocarpa (Vog.) Macbr. Monografia de especialização, Universidade Federal de Santa Maria, Santa Maria.

AULER, N. M. F., BATTISTIN, A. \& BIONDO, E. 1998. Análise do cariótipo de Apuleia leiocarpa (Vogel) Macbr. Genetics and Molecular Biology 21 (3-supplement):163.

BANDEL, G. 1974. Chromosome numbers and evolution in the Leguminosae. Caryologia 27:17-32.

BELTRÃA, G.T.A. \& GUERRA, M. 1990. Citogenética de angiospermas coletadas em Pernambuco - III. Ciência e Cultura 42:839-845.

BIONDO, E. 2004. Citotaxonomia e citogenética de espécies da subfamília Caesalpinioideae (Leguminosae) ocorrentes na região Sul do Brasil. Tese de doutorado, Universidade Federal do Rio Grande do Sul, Porto Alegre.

BORTOLUZZI, R.L.C. 2004. A subfamília Caesalpinioideae (Leguminosae) em Santa Catarina, Brasil. Tese de doutorado, Universidade Federal do Rio Grande do Sul, Porto Alegre.

BRUNEAU, A., FOREST, F., HERENDEEN, P. S., KLIGAARD, B.B. \& LEWIS, G.P. 2001. Phylogenetic relationships in the Caesalpinioideae (Leguminosae) as inferred from chloroplast trnL intron sequences. Systematic Botany 26:487-514.

BURKART, A. 1952. Las leguminosas argentinas silvestres y cultivadas. ACME Agency, Buenos Aires.

BURKART, A. 1987. Leguminosae. In Flora Ilustrada de Entre Rios (Argentina) (N.S.T. Burkart \& N.M. Bacigalupo, eds.). Buenos Aires, Colección Científica del I.N.T.A., v.6, p.695-704.

CASTRONOVO, A. 1945. Estudio cariológico de doce especies de leguminosas argentinas. Darwiniana 7:38-57.

COLEMAN, J.R. \& DEMENEZES, E.M. 1980. Chromosome numbers in Leguminosae from the state of São Paulo, Brazil. Rhodora 82:474-475.

CONCEIÇÃO, A.S., QUEIRÓZ, L.P. \& LEWIS, G.P. 2001. Novas espécies de Chamaecrista Moench (Leguminosae Caesalpinioideae) da Chapada Diamantina, Bahia, Brasil. Sitientibus série Ciências Biológicas 1:112-119.

COVAS, G. 1949. Estudios cariológicos en antófitas. Darwiniana 9:158-162.

DOYLE, J.J., DOYLE, J.L., BALLENGER, J.A., DICKSON,E.E., KAJITA, T. \& OHASHI, H. 1997. A phylogeny of the chloroplast gene rbcL in the Leguminosae: taxonomic correlations and insights into the evolution of nodulation. American Journal of Botany 84:541-554.
DOYLE, J.J., CHAPPILL, J.A., BEILEY, C.D. \& KAJITA, T. 2000. Towards a comprehensive phylogeny of legumes: evidence from rbcL sequences and non-molecular data. In Advances in Legume Systematics. (P.S. Herendeen \& A. Bruneau, eds.). Royal Botanic Gardens, Kew, part 9, p.45-64.

FEDEROV, A.A. 1969. Chromosome numbers of flowering plants. Russian Academy of Sciences. Leningrad.

FORNI-MARTINS, E.R. \& MARTINS, F.R. 2000. Chromosomes studies on Brazilian cerrado plants. Genetics and Molecular Biology 23:947-955.

FRAHM-LELIVELD, J.A. 1957. Observations cytologiques sur quelques Légumineuses tropicales et subtropicales. Revue de Cytologie et de Biologie Végétales le Botaniste 18:273-292.

GHAREEB, A., KHALIFA, S.F. \& FAWZI, N. 1999. Molecular systematics of some Cassia species. Cytologia 64:11-16.

GIBBS, P.E. \& INGRAM, R. 1982. Chromosome numbers of some Brazilian flowering plants. Notes from the Royal Botanic Garden Edinburgh 40:399-407.

GILL, L.S. \& HUSAINI, S.W.H. 1985. Caryological evolution of the southern Nigerian Leguminosae. Revue de Cytologie et de Biologie Végétales le Botaniste 8:3-31.

GOLDBLATT, P. 1981a. Cytology and the phylogeny of Leguminosae. In Advances in Legume Systematics (R.M. Polhill \& P.H. Raven, eds.). Royal Botanical Gardens, Kew, part 2, p.427- 463.

GOLDBLATT, P. 1981b. Chromosome numbers in Legumes II. Annals of the Missouri Botanical Garden 68:551-557.

GOLDBLATT, P. 1985. Index to plant chromosome numbers 1982-1983. Monographs in Systematic Botany from the Missouri Botanical Garden 13:1-224.

GOLDBLATT, P. 1988. Index to plant chromosome numbers 1984-1985. Monographs in Systematic Botany from the Missouri Botanical Garden 23:1-264.

GOLDBLATT, P. \& JOHNSON, D.E. 1990. Index to plant chromosome numbers 1986-1987. Monographs in Systematic Botany from the Missouri Botanical Garden 30:1-243.

GOLDBLATT, P. \& JOHNSON, D.E. 1991. Index to plant chromosome numbers 1988-1989. Monographs in Systematic Botany from the Missouri Botanical Garden 40:1-238.

GOLDBLATT, P. \& JOHNSON, D.E. 1994. Index to plant chromosome numbers 1990-1991. Monographs in Systematic Botany from the Missouri Botanical Garden 51:1-267.

GOLDBLATT, P. \& JOHNSON, D.E. 1996. Index to plant chromosome numbers 1992-1993. Monographs in Systematic Botany from the Missouri Botanical Garden 58:1-276.

GOLDBLATT, P. \& JOHNSON, D.E. 1998. Index to plant chromosome numbers 1994-1995. Monographs in Systematic Botany from the Missouri Botanical Garden 69:1-208. 
GOLDBLATT, P. \& JOHNSON, D.E. 2000. Index to plant chromosome numbers 1996-1997. Monographs in Systematic Botany from the Missouri Botanical Garden 81:1-188.

GUERRA, M. 1990. A situação da citotaxonomia de Angiospermas nos trópicos e, em particular, no Brasil. Acta Botanica Brasilica 4:75-86.

GUERRA, M. 2000. Chromosome number variation and evolution in monocots. In Monocots systematics and evolution. (K.L. Wilson \& D.A. Morrison, eds.). CSIRO, Melbourne, p.127-136.

HERENDEEN, P.S. 2000. Structural evolution in the Caesalpinioideae (Leguminosae). In Advances in Legume Systematics (P.S. Herendeen \& A. Bruneau, eds.). Royal Botanical Gardens, Kew, part 9, p.45-64.

IRWIN, H.S. 1964. Monographic studies in Cassia (Leguminosae - Caesalpinioideae) I. Section Xerocalyx. Memoirs of the New York Botanical Garden 12:1-114.

IRWIN, H.S. \& BARNEBY, R.C. 1981. Cassieae. In Advances in Legumes Systematics (R.M. Polhill \& P.H. Raven, eds.). Royal Botanic Gardens, Kew, part 1, p.97-106.

IRWIN, H.S. \& BARNEBY, R.C. 1982. The American Cassiinae, a synoptical revision of Leguminosae Tribe Cassieae, subtribe Cassiinae in the New World. Memoirs of the New York Botanical Garden 35:1-918.

IRWIN, H.S. \& TURNER, B.L. 1960. Chromosomal relationships and taxonomics considerations in the genus Cassia. American Journal of Botany 47:309-318.

JAHAN, B., VAHIDY, A.A. \& ALI, S.I. 1994. Chromosome numbers in some taxa of Fabaceae mostly native to Pakistan. Annals of the Missouri Botanical Garden 81:792-799.

KAJITA, T., OHASHI, H., TATEISHI, Y., BAILEY, C.D. \& DOYLE, J.J. 2001. RbcL and legume phylogeny, with particular reference to Phaseoleae, Millettieae, and allies. Systematic Botany 26:515-536.

K ̈̈SS, E. \& WINK, M. 1996. Molecular evolution of the Leguminosae: phylogeny of the three subfamilies based on rbcL-sequences. Biochemical Systematics and Ecology 24:365-378.

KUMARI, S. \& BIR, S.S. 1989. Karyomorphological evolution in Caesalpiniaceae. Journal of Cytology \& Genetics 24:149-163.

LEWIS, G.P. 1987. Legumes of Bahia. Royal Botanic Gardens, Kew.

LEWIS, G.P. \& POLHILL, R.M. 1998. A situação atual da sistemática de Leguminosae neotropicais. Monographs in Systematic Botany from the Missouri Botanical Garden 68:113-129.

LEWIS, G.P., SIMPSON, B.B. \& NEFF, J.L. 2000. Progress in understanding the reproductive biology of the Caesalpinioideae (Leguminosae). In Advances in Legume Systematics (P.S. Herendeen \& A. Bruneau, eds.). Royal Botanic Gardens, Kew, part 9, p.45-64.

MIÉGE, J. 1960. Troisiéme liste de nombres chromosomiques d'espéces d'Afrique occidentale. Annales de la Faculté de Sciences de l'Université de Dakar 5:75-76.
OHRI, D., KUMAR, A. \& PAL, M. 1986. Correlations between 2C DNA values in habit in Cassia (Leguminosae: Caesalpinioideae). Plant Systematics and Evolution 153:223-227.

ORMOND, W.T., CORREIA, M.C.R. \& OLIVEIRA, R.F. 1977. Contribuição à citologia de Cassia tetraphylla Desvaux. I: Número de cromossomas. Revista Brasileira de Biologia 37:717-720.

PEDROSA,A., GITAÍ, J., SILVA,A.E.B., FELIX, L.P. \& GUERRA, M. 1999. Citogenética de angiospermas coletadas em Pernambuco - V. Acta Botanica Brasilica 13:49-60.

POLHILL, R.M. 1994. Classification of the Leguminosae. In Phytochemical Dictionary of the Leguminosae. (F.A. Bisby, J. Buckingham \& J.B. Harborne, eds.). Chapman \& Hall, London, v.1, p.35-54.

POLHILL, R.M., RAVEN, P.H. \& STIRTON, C.H. 1981. Evolution and systematics of the Leguminosae. In Advances in Legumes Systematics. (R.M. Polhill \& P.H. Raven, eds.). Royal Botanic Gardens, Kew, part 1, p.1-26.

RAVEN, P.H. \& POLHILL, R.M. 1981. Biogeography of the Leguminosae. In Advances in Legumes Systematics (R.M. Polhill \& P.H. Raven, eds.). Royal Botanic Gardens, Kew, part 1, p.27-34.

RIBEIRO, J.E.L.S., HOPKINS, M.J.C. \& VICENTINI, A. 1999. Flora da Reserva Ducke: guia de identificação das plantas vasculares de uma floresta de terra-firme na Amazônia Central. INPA, Manaus, p.382-395.

SIMPSON, B.B. \& LEWIS, G.P. 2003. New combinations in Pomaria (Caesalpinioideae: Leguminosae). Kew Bulletin 58:175-184.

SOUZA, M.G.C. 2000. Citogenética de Leguminosae da Amazônia Oriental, Pará. Dissertação de mestrado, Universidade Federal de Pernambuco, Recife.

SOUZA, M.G.C. \& BENKO-ISEPPON, A.M. 2004. Cytogenetics and chromosome banding patterns in Caesalpinioideae and Papilionoideae species of Pará, Amazonas, Brazil. Botanical Journal of the Linnean Society 144:181-191.

SOUZA, M.G.C., BENKO-ISEPPON, A.M., FERREIRA, G. \& VIANA, R.C.M. 2000. Cytogenetics of Leguminosae: report of 12 species from Amazonian Rain Forest in Pará (Brazil). Genetics and Molecular Biology 23 (3 - supplement):379.

TUCKER, S.C. 1996. Trends in evolution of floral ontogeny in Cassia sensu stricto, Senna, and Chamaecrista (Leguminosae: Caesalpinioideae: Cassieae: Cassiinae); a study in convergence. American Journal of Botany 83:687-711.

TUCKER, S.C. \& DOUGLAS, A.W. 1994. Ontogenetic evidence and phylogenetic relationships among basal taxa of legumes. In Advances in Legume Systematics (I.K. Ferguson \& S.C. Tucker, eds.). Royal Botanic Gardens, Kew, part 6, p.11-32.

TURNER, B.L. 1956. Chromosome numbers in the Leguminosae. I. American Journal of Botany 43:577-581. 